\title{
Doubly universal Taylor series on simply connected domains
}

\author{
Nicky Chatzigiannakidou' ${ }^{1}$ - Vagia Vlachou ${ }^{1}$
}

Received: 12 April 2016 / Revised: 16 July 2016 / Accepted: 18 July 2016 /

Published online: 3 August 2016

C) Springer International Publishing AG 2016

\begin{abstract}
We deal with the existence of doubly universal Taylor series defined on simply connected domains with respect to any center and generalize the results of Costakis and Tsirivas for the unit disk.
\end{abstract}

Keywords Universal Taylor series · Doubly universality · Disjoint hypercyclicity · Degree of approximation

Mathematics Subject Classification 47A16 · 30K05 $\cdot 41 \mathrm{~A} 10$

\section{Introduction}

Let $\Omega \subset \mathbb{C}$ be an open set. We denote by $H(\Omega)$ the space of functions, holomorphic in $\Omega$, endowed with the topology of uniform convergence on compacta. Moreover, for a compact set $K \subset \mathbb{C}$, we denote

$$
\mathcal{A}(K)=\left\{g \in H\left(K^{\mathrm{o}}\right): g \text { is continuous on } K\right\}
$$

and

$$
\mathcal{M}=\left\{K \subset \mathbb{C}: K \text { is a compact set and } K^{\mathrm{c}} \text { is a connected set }\right\}
$$

$\triangle$ Vagia Vlachou

vvlachou@math.upatras.gr

Nicky Chatzigiannakidou

ni.chatzig@gmail.com

1 Department of Mathematics, University of Patras, University Campus, 26500 Patras, Greece 
Let $\zeta_{0} \in \Omega$. It is well known that a function $f \in H(\Omega)$ has a Taylor expansion around $\zeta_{0}$, which is valid inside the disk of convergence.

If we denote by $S_{N}\left(f, \zeta_{0}\right)(z)=\sum_{n=0}^{N} f^{(n)}\left(\zeta_{0}\right)\left(z-\zeta_{0}\right)^{n} / n !, N=1,2, \ldots, z \in$ $\mathbb{C}$, the partial sums of the Taylor expansion of $f$ around $\zeta_{0}$, then this sequence of polynomials may have very strong approximation properties outside $\Omega$. To be more specific, let us recall the definition of universal Taylor series due to Nestoridis (see [5, 6]): A function $f \in H(\Omega)$ is said to belong to the collection $U\left(\Omega, \zeta_{0}\right)$ of functions with universal Taylor series expansions around $\zeta_{0}$ if the partial sums $\left\{S_{N}\left(f, \zeta_{0}\right)\right.$ : $N=1,2, \ldots\}$ are dense in $A(K)$, for every $K \in \mathcal{M}$ disjoint from $\Omega$ (the topology of $A(K)$ is induced by the norm $\left.\|g\|_{K}=\max _{z \in K}|g(z)|\right)$. Nestoridis [5,6] has shown that $U\left(\Omega, \zeta_{0}\right) \neq \varnothing$, for any simply connected domain $\Omega$ and any point $\zeta_{0} \in \Omega$. In particular, he showed that the collection $U\left(\Omega, \zeta_{0}\right)$ is a dense, $G_{\delta}$ subset of the space $H(\Omega)$.

Recently, Costakis and Tsirivas in [3] worked with $\Omega=\mathbb{D}$ (open unit disk) and introduced the following stronger notion of universality with respect to Taylor series.

Definition 1.1 Let $\left(\lambda_{n}\right)_{n \in \mathbb{N}}$ be a strictly increasing sequence of positive integers. We say that a function $f \in H(\mathbb{D})$ belongs to the class $U_{\mathrm{CT}}\left(\mathbb{D},\left(\lambda_{n}\right)\right)$ if the set $\left\{\left(S_{n}(f, 0)(z), S_{\lambda_{n}}(f, 0)(z)\right): n=1,2, \ldots\right\}$ is dense in $A(K) \times A(K)$, for every $K \in \mathcal{M}$ disjoint from $\mathbb{D}$. Such a function $f$ is called doubly universal Taylor series with respect to the sequences $(n)$ and $\left(\lambda_{n}\right)$.

Costakis and Tsirivas proved that the class $U_{\mathrm{CT}}\left(\mathbb{D},\left(\lambda_{n}\right)\right)$ is non-empty if and only if $\lim \sup _{n \in \mathbb{N}} \lambda_{n} / n=+\infty$. We shall study a class of functions with even stronger approximation properties. Namely,

Definition 1.2 Let $\left(\lambda_{n}\right)_{n \in \mathbb{N}}$ be a strictly increasing sequence of positive integers, $\Omega$ a simply connected domain and $\zeta_{0} \in \Omega$. We say that a function $f \in H(\Omega)$ belongs to the class $U_{\mathrm{CV}}\left(\Omega,\left(\lambda_{n}\right), \zeta_{0}\right)$ if $\left\{\left(S_{n}\left(f, \zeta_{0}\right)(z), S_{\lambda_{n}}\left(f, \zeta_{0}\right)(z)\right): n=1,2, \ldots\right\}$ is dense in $A\left(K_{1}\right) \times A\left(K_{2}\right)$, for every pair of sets $K_{1}, K_{2} \in \mathcal{M}$ disjoint from $\Omega$.

Our main result is again that the class $U_{\mathrm{CV}}\left(\Omega,\left(\lambda_{n}\right)\right)$ is non-empty if and only if $\lim \sup _{n \in \mathbb{N}} \lambda_{n} / n=+\infty$. We extend the method used in [3] in order to obtain the result for different compact sets $K_{1}, K_{2}$ and we also overcome some technical difficulties to generalize it to any simply connected domain $\Omega$.

Analogous results for other universality phenomena (e.g. universality with respect to derivatives or translations) can be found in $[1,2]$. The article of Costakis and Tsirivas has complete bibliography on the subject, which features the motivation for investigating such questions.

\section{Positive result}

Let $K, L$ be compact sets and $f: K \rightarrow \mathbb{C}$ be a continuous function defined on $K$. Let $p$ be a polynomial. We denote by $\operatorname{deg} p$ the degree of the polynomial, i.e. the highest degree of terms with non-zero coefficients and by ldeg $p$ the lowest degree of terms with non-zero coefficients. Then for any choice of integers $n>m>1$ we use the notation 


$$
\begin{aligned}
d_{n, m}(f, K, L)=\inf \{\max \{ & \left.\|f-p\|_{K},\|p\|_{L}\right\}: \\
p & \text { is a polynomial such that } \operatorname{ldeg} p \geqslant m \text { and } \operatorname{deg} p \leqslant n\} .
\end{aligned}
$$

The following proposition is crucial for our result. It is a slight modification of the corresponding proposition in [3].

Proposition 2.1 Let $K, L \in \mathcal{M}$ be two disjoint compact sets such that $0 \in L^{\mathrm{o}}$. Let, in addition, $\left(\tau_{n}\right)_{n \in \mathbb{N}}$ and $\left(\sigma_{n}\right)_{n \in \mathbb{N}}$ be two sequences of positive integers such that $\tau_{n} / \sigma_{n} \rightarrow+\infty$ and $U \subset \mathbb{C}$ be open, $K \subset U$. Then there exits $\theta \in(0,1)$ such that for every function $f$ holomorphic in $U$

$$
\limsup _{n \in \mathbb{N}} d_{\tau_{n}, \sigma_{n}}(f, K, L)^{1 / \tau_{n}}<\theta .
$$

Its proof is similar to the proof of [3, Theorem 2.1] and we omit it.

Theorem 2.2 Let $\Omega$ be a simply connected domain and $\zeta_{0} \in \Omega$. Consider a strictly increasing sequence of positive integers $\left(\lambda_{n}\right)_{n \in \mathbb{N}}$ such that $\lim \sup _{n \in \mathbb{N}} \lambda_{n} / n=+\infty$. Then the set $U_{\mathrm{CV}}\left(\Omega,\left(\lambda_{n}\right), \zeta_{0}\right)$ is $G_{\delta}$ and dense in $H(\Omega)$.

Proof Let $\left(f_{j}\right)_{j \in \mathbb{N}}$ be an enumeration of all polynomials with coefficients in $\mathbb{Q}+i \mathbb{Q}$ and let $\left(K_{m}\right)_{m \in \mathbb{N}}$ be a sequence of sets in $\mathcal{M}$ disjoint from $\Omega$ such that the following holds: every non-empty compact set $K \subset \mathbb{C} \backslash \Omega$, having connected complement, is contained in some $K_{m}$ (for the existence of such a sequence we refer to [6]).

For any positive integers $m_{1}, m_{2}, j_{1}, j_{2}, s, n$, we denote by $E\left(m_{1}, m_{2}, j_{1}, j_{2}, s, n\right)$ the set

$$
\begin{aligned}
E\left(m_{1}, m_{2}, j_{1}, j_{2}, s, n\right)=\left\{f \in H(\Omega):\left\|S_{n}\left(f, \zeta_{0}\right)-f_{j_{1}}\right\|_{K_{m_{1}}}<\frac{1}{s}\right. & \\
& \text { and } \left.\left\|S_{\lambda_{n}}\left(f, \zeta_{0}\right)-f_{j_{2}}\right\|_{K_{m_{2}}}<\frac{1}{s}\right\} .
\end{aligned}
$$

Using Mergelyan's theorem, it is easy to prove that

$$
U_{\mathrm{CV}}\left(\Omega,\left(\lambda_{n}\right), \zeta_{0}\right)=\bigcap_{m_{1}, m_{2}, j_{1}, j_{2}, s \in \mathbb{N}} \bigcup_{n \in \mathbb{N}} E\left(m_{1}, m_{2}, j_{1}, j_{2}, s, n\right)
$$

(see for example a similar proof in [6]). Moreover, for every $m_{1}, m_{2}, j_{1}, j_{2}, s, n \in \mathbb{N}$, the set $E\left(m_{1}, m_{2}, j_{1}, j_{2}, s, n\right)$ is open (again, see for example [6]).

Therefore, in view of Baire's Category theorem, it suffices to prove that, for every choice of positive integers $m_{1}, m_{2}, j_{1}, j_{2}$ and $s$, the set $\bigcup_{n \in \mathbb{N}} E\left(m_{1}, m_{2}, j_{1}, j_{2}, s, n\right)$ is dense in $H(\Omega)$.

Fix $m_{1}, m_{2}, j_{1}, j_{2}$ and $s \in \mathbb{N}$. Let $\varepsilon>0, L \subset \Omega$ be a compact set and $g \in H(\Omega)$. Without loss of generality, we may assume that $\zeta_{0} \in L^{0}$ and that the compact set $L$ has connected complement (note that $\Omega$ is simply connected). We will prove the existence of a function $f \in H(\Omega)$ and $n \in \mathbb{N}$ such that: 
- $\sup _{z \in L}|f(z)-g(z)|<\varepsilon$,

- $\sup _{z \in K_{m_{1}}}\left|S_{n}\left(f, \zeta_{0}\right)(z)-f_{j_{1}}(z)\right|<1 / s$,

- $\sup _{z \in K_{m_{2}}}\left|S_{\lambda_{n}}\left(f, \zeta_{0}\right)(z)-f_{j_{2}}(z)\right|<1 / s$.

First, we apply Runge's theorem to find a polynomial $p$ such that $\sup _{z \in L} \mid p(z)-$ $g(z) \mid<\varepsilon / 2$ and $\sup _{z \in K_{m_{1}}}\left|p(z)-f_{j_{1}}(z)\right|<1 / s$.

Since $\lim \sup _{n \in \mathbb{N}} \lambda_{n} / n=+\infty$, there exists a strictly increasing sequence of positive integers $\left(\mu_{n}\right)_{n \in \mathbb{N}}$ such that $\lambda_{\mu_{n}} / \mu_{n} \rightarrow+\infty$. Thus, $\lambda_{\mu_{n}} /\left(\mu_{n}+1\right) \rightarrow+\infty$ as $n \rightarrow+\infty$. It is easy to see that the sets $K_{m_{2}}-\zeta_{0}=\left\{z-\zeta_{0}: z \in K_{m_{2}}\right\}$ and $L-\zeta_{0}=\left\{z-\zeta_{0}: z \in L\right\}$ are compact, disjoint and they have connected complements. Applying Proposition 2.1, we have

$$
\limsup _{n \in \mathbb{N}}\left[d_{\lambda_{\mu_{n}}, \mu_{n}+1}\left(f_{j_{2}}\left(z+\zeta_{0}\right)-p\left(z+\zeta_{0}\right), K_{m_{2}}-\zeta_{0}, L-\zeta_{0}\right)\right]^{1 / \lambda \mu_{n}}<\theta,
$$

for some $\theta \in(0,1)$. Therefore, there exists $N \in \mathbb{N}$ such that

$$
\left[d_{\lambda_{\mu_{n}}, \mu_{n}+1}\left(f_{j_{2}}\left(z+\zeta_{0}\right)-p\left(z+\zeta_{0}\right), K_{m_{2}}-\zeta_{0}, L-\zeta_{0}\right)\right]^{1 / \lambda_{\mu_{n}}}<\theta, \quad n \geqslant N .
$$

Thus, we may choose a sequence of polynomials $P_{n}, n \geqslant N$, with ldeg $P_{n} \geqslant \mu_{n}+1$ and $\operatorname{deg} P_{n} \leqslant \lambda_{\mu_{n}}$ such that

$$
\sup _{z \in K_{m_{2}}-\zeta_{0}}\left|f_{j_{2}}\left(z+\zeta_{0}\right)-p\left(z+\zeta_{0}\right)-P_{n}(z)\right| \leqslant \theta^{\lambda_{\mu_{n}}} \quad \text { and } \sup _{z \in L-\zeta_{0}}\left|P_{n}(z)\right| \leqslant \theta^{\lambda_{\mu_{n}}} .
$$

Let us fix $n_{0} \in \mathbb{N}, n_{0} \geqslant N$, such that $\mu_{n_{0}} \geqslant \operatorname{deg} p$ and $\theta^{\lambda \mu_{n_{0}}}<\min \{\varepsilon / 2,1 / s\}$. We set $f(z)=P_{n_{0}}\left(z-\zeta_{0}\right)+p(z)$. Then $S_{\mu_{n_{0}}}\left(f, \zeta_{0}\right)(z)=S_{\mu_{n_{0}}}\left(p, \zeta_{0}\right)(z)=p(z)$. Also, $S_{\lambda_{\mu_{n_{0}}}}\left(f, \zeta_{0}\right)(z)=f(z)$. Thus,

$$
\begin{aligned}
& \sup _{z \in L}|f(z)-g(z)|=\sup _{z \in L}\left|P_{n_{0}}\left(z-\zeta_{0}\right)+p(z)-g(z)\right| \\
& \leqslant \sup _{z \in L}\left|P_{n_{0}}\left(z-\zeta_{0}\right)\right|+\sup _{z \in L}|p(z)-g(z)| \\
& =\sup _{z \in L-\zeta_{0}}\left|P_{n_{0}}(z)\right|+\sup _{z \in L}|p(z)-g(z)| \\
& \leqslant \theta^{\lambda_{\mu_{n}}}+\frac{\varepsilon}{2}<\varepsilon, \\
& \sup _{z \in K_{m_{1}}}\left|S_{\mu_{n_{0}}}\left(f, \zeta_{0}\right)(z)-f_{j_{1}}(z)\right|=\sup _{z \in K_{m_{1}}}\left|p(z)-f_{j_{1}}(z)\right|<\frac{1}{s}, \\
& \sup _{z \in K_{m_{2}}}\left|S_{\lambda_{\mu_{n}}}\left(f, \zeta_{0}\right)(z)-f_{j_{2}}(z)\right|=\sup _{z \in K_{m_{2}}}\left|P_{n_{0}}\left(z-\zeta_{0}\right)+p(z)-f_{j_{2}}(z)\right| \\
& =\sup _{z \in K_{m_{2}}-\zeta_{0}}\left|P_{n_{0}}(z)+p\left(z+\zeta_{0}\right)-f_{j_{2}}\left(z+\zeta_{0}\right)\right| \\
& \leqslant \theta^{\lambda \mu_{n_{0}}}<\frac{1}{S} \text {. }
\end{aligned}
$$

The function $f$ satisfies all the requirements and the result follows. 


\section{Negative result}

In this section we shall use the notation $U_{\mathrm{CT}}\left(\Omega,\left(\lambda_{n}\right)\right)$ for the corresponding class of Costakis and Tsirivas in any simply connected domain $\Omega$ (i.e. in Definition 1.2, we assume that $\left.K_{1}=K_{2}\right)$. Obviously $U_{\mathrm{CV}}\left(\Omega,\left(\lambda_{n}\right)\right) \subset U_{\mathrm{CT}}\left(\Omega,\left(\lambda_{n}\right)\right)$. So, in order to prove that the class $U_{\mathrm{CV}}\left(\Omega,\left(\lambda_{n}\right)\right)$ is empty if $\lim _{\sup } \operatorname{se}_{n} \lambda_{n} / n \neq+\infty$, we shall prove a stronger result that the class $U_{\mathrm{CT}}\left(\Omega,\left(\lambda_{n}\right)\right)$ is in this case empty. For this we need the following result of Müller and Yavrian in [4].

Theorem 3.1 (Müller-Yavrian) Let $\Gamma$ be a compact and connected subset of $\mathbb{C}$, but not a singleton. Let $E \subset \mathbb{C}$ be a closed set such that $E$ is non-thin at $\infty$. Also suppose $\left(P_{n}\right)_{n \in \mathbb{N}}$ is a sequence of polynomials with $\operatorname{deg} P_{n} \leqslant d_{n}$, for some increasing sequence of positive integers $\left(d_{n}\right)_{n \in \mathbb{N}}$, and having the following properties:

- there exists a function $f: \Gamma \rightarrow \mathbb{C}$ with $\lim _{\lim } \sup _{n \rightarrow+\infty}\left\|f-P_{n}\right\|_{\Gamma}^{1 / d_{n}}<1$,

- for all $z \in E$, lim $\sup _{n \rightarrow+\infty}\left|P_{n}(z)\right|^{1 / d_{n}} \leqslant 1$.

Then the following statements are true:

(i) If the sequence $\left(d_{n+1} / d_{n}\right)_{n \in \mathbb{N}}$ is bounded, then $f$ extends to an entire function and, for every compact set $K \subset \mathbb{C}$, we have lim $\sup _{n \rightarrow+\infty}\left\|f-P_{n}\right\|_{K}^{1 / d_{n}}<1$.

(ii) If, for an arbitrary $\left(d_{n}\right)_{n \in \mathbb{N}}$, the function $f$ is analytic on $\Gamma$, then $f$ extends to a holomorphic function having a simply connected domain of existence $G_{f} \subset \mathbb{C}$ $\left(G_{f}\right.$ denotes the unique largest domain on which $f$ extends as a holomorphic function; observe that $G_{f}$ exists in this case) and, for every compact set $K \subset G_{f}$, we have $\lim \sup _{n \rightarrow+\infty}\left\|f-P_{n}\right\|_{K}^{1 / d_{n}}<1$.

Theorem 3.2 Let $\Omega \subset \mathbb{C}$ be a simply connected domain and $\zeta_{0} \in \Omega$. If $\left(\lambda_{n}\right)_{n \in \mathbb{N}}$ is a strictly increasing sequence of positive integers such that $\lim _{\sup _{n} \in \mathbb{N}} \lambda_{n} / n<+\infty$, then $U_{\mathrm{CT}}\left(\Omega,\left(\lambda_{n}\right), \zeta_{0}\right)=\varnothing$.

Proof Assume first that $\Omega$ is not bounded. Arguing by contradiction, suppose that there exists $f \in U_{\mathrm{CT}}\left(\Omega,\left(\lambda_{n}\right), \zeta_{0}\right)$. Since the sequence $\left(\lambda_{n} / n\right)_{n \in \mathbb{N}}$ is bounded, there exists a positive integer $C$ such that $\lambda_{n} / n<C$, for every $n \in \mathbb{N}$.

Let $R>0$ be the radious of convergence of the Taylor series of $f$ around $\zeta_{0}$. Fix a positive number $a>\max \left\{R^{1 / C}, 1\right\}$. Consider the sets

$$
E_{n}=\Omega^{\mathrm{c}} \cap D\left(\zeta_{0}, a^{C}\right)^{\mathrm{c}} \cap \overline{D\left(\zeta_{0}, a^{C}+n\right)}, \quad n=1,2, \ldots
$$

(we denote by $D(z, r)$ the open disk of center $z$ and radius $r$ ). Without loss of generality, we may assume that $E_{n} \neq \varnothing, n \geqslant 1$, since this is eventually the case anyway (or by choosing a suitable positive integer $C$ ).

For every $n \in \mathbb{N}$, it is easy to see that sets $E_{n} \subset \Omega^{\mathrm{c}}$ are closed and bounded, thus compact. Moreover, each $E_{n}$ has connected complement. To see this, note that $E_{n}^{\mathrm{c}}=$ $\Omega \cup D\left(\zeta_{0}, a^{C}\right) \cup \overline{D\left(\zeta_{0}, a^{C}+n\right)^{c}}$ and $\Omega, D\left(\zeta_{0}, a^{C}\right)$ and $\overline{D\left(\zeta_{0}, a^{C}+n\right)^{c}}$ are connected sets. Furthermore, $\zeta_{0} \in \Omega \cap D\left(\zeta_{0}, a^{C}\right), \Omega$ is unbounded and the set $\overline{D\left(\zeta_{0}, a^{C}+n\right)^{c}}$ is a neighborhood of infinity. 
Let $E=\bigcup_{n \in \mathbb{N}} E_{n}=\Omega^{\mathrm{c}} \cap D\left(\zeta_{0}, a^{C}\right)^{\mathrm{c}}$. Since $\Omega^{\mathrm{c}}$ is connected, it is non-thin at $\infty$ (see [7, Theorem 3.8.3, p. 79]). As thinness is a local property (see a definition on [7, p. 79]), $E$ is also non-thin at $\infty$.

Now, we fix a point $\xi \in \partial \Omega$ with $\left|\xi-\zeta_{0}\right|=R$. Since $f$ belongs to the class $U_{\mathrm{CT}}\left(\Omega,\left(\lambda_{n}\right), \zeta_{0}\right)$, we may fix $n_{1} \in \mathbb{N}$ such that

$$
\sup _{z \in E_{1} \cup\{\xi\}}\left|S_{n_{1}}\left(f, \zeta_{0}\right)(z)\right|<\frac{1}{2 a^{C}}, \quad \sup _{z \in E_{1} \cup\{\xi\}}\left|S_{\lambda_{n_{1}}}\left(f, \zeta_{0}\right)(z)-1\right|<\frac{1}{2 a^{C}} .
$$

Thus,

$$
\begin{aligned}
& \sup _{z \in E_{1} \cup\{\xi\}}\left|S_{\lambda_{n_{1}}}\left(f, \zeta_{0}\right)(z)-S_{n_{1}}\left(f, \zeta_{0}\right)(z)-1\right| \\
& \quad \leqslant \sup _{z \in E_{1} \cup\{\xi\}}\left|S_{n_{1}}\left(f, \zeta_{0}\right)(z)\right|+\sup _{z \in E_{1} \cup\{\xi\}}\left|S_{\lambda_{n_{1}}}\left(f, \zeta_{0}\right)(z)-1\right|<\frac{1}{a^{C}} .
\end{aligned}
$$

For every $z \in \mathbb{C} \backslash\left\{\zeta_{0}\right\}$, we set

$$
\frac{R^{n_{1}}}{\left(z-\zeta_{0}\right)^{n_{1}}}\left[S_{\lambda_{n_{1}}}\left(f, \zeta_{0}\right)(z)-S_{n_{1}}\left(f, \zeta_{0}\right)(z)\right]=P_{1}(z)
$$

Then $P_{1}$ is extended as a polynomial with $\operatorname{deg} P_{1} \leqslant \lambda_{n_{1}}-n_{1}=n_{1}\left(\lambda_{n_{1}} / n_{1}-1\right) \leqslant$ $n_{1}(C-1)$. Moreover,

$$
\sup _{z \in E_{1} \cup\{\xi\}}\left|\frac{\left(z-\zeta_{0}\right)^{n_{1}}}{R^{n_{1}}} P_{1}(z)-1\right|<\frac{1}{a^{C}} .
$$

Finally, since $z \in E_{1}$ implies that $\left|z-\zeta_{0}\right|>a^{C}$ we have

$$
\begin{aligned}
\sup _{z \in E_{1}}\left|P_{1}(z)\right| & \leqslant \sup _{z \in E_{1}} \frac{R^{n_{1}}}{\left|\left(z-\zeta_{0}\right)^{n_{1}}\right|} \sup _{z \in E_{1}}\left|\frac{\left(z-\zeta_{0}\right)^{n_{1}}}{R^{n_{1}}} P_{1}(z)\right| \\
& \leqslant\left(\frac{R}{a^{C}}\right)^{n_{1}}\left(\sup _{z \in E_{1}}\left|\frac{\left(z-\zeta_{0}\right)^{n_{1}}}{R^{n_{1}}} P_{1}(z)-1\right|+1\right) \\
& <\left(\frac{R}{a^{C}}\right)^{n_{1}}\left(\frac{1}{a^{C}}+1\right)<2\left(\frac{R}{a^{C}}\right)^{n_{1}} .
\end{aligned}
$$

Repeating the above argument, we fix $n_{2} \in \mathbb{N}$, with $n_{2}>n_{1}$, such that

$$
\sup _{z \in E_{2} \cup\{\xi\}}\left|S_{n_{2}}\left(f, \zeta_{0}\right)(z)\right|<\frac{1}{3 a^{C}}, \quad \sup _{z \in E_{2} \cup\{\xi\}}\left|S_{\lambda_{n_{2}}}\left(f, \zeta_{0}\right)(z)-1\right|<\frac{1}{3 a^{C}} .
$$

As before, we set

$$
\frac{R^{n_{2}}}{\left(z-\zeta_{0}\right)^{n_{2}}}\left[S_{\lambda_{n_{2}}}\left(f, \zeta_{0}\right)(z)-S_{n_{2}}\left(f, \zeta_{0}\right)(z)\right]=P_{2}(z)
$$


Then $P_{2}$ is an extended polynomial of degree less than $n_{2}(C-1)$. Moreover, we have

$$
\sup _{z \in E_{2} \cup\{\xi\}}\left|\frac{\left(z-\zeta_{0}\right)^{n_{2}}}{R^{n_{2}}} P_{2}(z)-1\right|<\frac{2}{3 a^{C}}
$$

and

$$
\sup _{z \in E_{2}}\left|P_{2}(z)\right|<2\left(\frac{R}{a^{C}}\right)^{n_{2}}
$$

Proceeding inductively, we conclude that for every $k \in \mathbb{N}$, there exists $n_{k} \in \mathbb{N}$, with $n_{k}>n_{k-1}$, and a polynomial $P_{k}$, deg $P_{k} \leqslant C n_{k}$, such that

$$
\sup _{z \in E_{k} \cup\{\xi\}}\left|\frac{\left(z-\zeta_{0}\right)^{n_{k}}}{R^{n_{k}}} P_{k}(z)-1\right|<\frac{2}{(k+1) a^{C}}
$$

and $\sup _{z \in E_{k}}\left|P_{k}(z)\right| \leqslant 2\left(R / a^{C}\right)^{n_{k}}$. Let $z \in E$, then

$$
\limsup _{k \rightarrow \infty}\left|P_{k}(z)\right|^{1 / C n_{k}} \leqslant \lim _{k \rightarrow \infty} 2^{1 / C n_{k}} \frac{R^{1 / C}}{a}=\frac{R^{1 / C}}{a}<1 .
$$

(Note that $\left(E_{n}\right)_{n \in \mathbb{N}}$ is an increasing sequence of compact sets.) have

Also, since $\sup _{z \in E_{1}}\left|P_{k}(z)\right| \leqslant \sup _{z \in E_{k}}\left|P_{k}(z)\right| \leqslant 2\left(R / a^{C}\right)^{n_{k}}$, for every $k \in \mathbb{N}$, we

$$
\limsup _{k \rightarrow \infty}\left\|P_{k}(z)\right\|_{E_{1}}^{1 / C n_{k}} \leqslant \frac{R^{1 / C}}{a}<1 .
$$

Therefore, we may apply the Müller-Yavrian theorem to a compact and connected subset $\Gamma$ of $E_{1}$ (containing more than one point), the closed set $E$ which is non-thin at $\infty$, the sequence of polynomials $P_{k}, d_{k}=C n_{k}, k \in \mathbb{N}$, and the function $f \equiv 0$. From inequalities (2), (3), we observe that the conditions of the theorem are fulfilled. Since the function $f \equiv 0$ is entire, it follows that $P_{k} \rightarrow 0$ uniformly on all compact subsets of $\mathbb{C}$. In particular, $P_{k}(\xi) \rightarrow 0$ as $k \rightarrow \infty$. On the other hand, (1) implies that $\left(\xi-\zeta_{0}\right)^{n_{k}} P_{k}(\xi) / R^{n_{k}} \rightarrow 1$ as $k \rightarrow \infty$, which is a contradiction. As a result, in case when $\Omega$ is not bounded, the class $U_{\mathrm{CT}}\left(\Omega,\left(\lambda_{n}\right), \zeta_{0}\right)$ is empty.

Now, if the set $\Omega$ is bounded, then $\Omega \cup D\left(\zeta_{0}, a^{C}\right) \subset D(0, N)$, for some $N \in \mathbb{N}$. One can apply the previous procedure for the compact sets $E_{n}=[N+1, N+n]$, for $n>1$ and the result follows.

Acknowledgments We would like to thank Georgios Costakis and Nikos Tsirivas for sharing with us the unpublished version of their work and thus informing us about these nice problems.

\section{References}

1. Bernal-González, L.: Disjoint hypercyclic operators. Studia Math. 182(2), 113-131 (2007)

2. Bès, J., Peris, A.: Disjointness in hypercyclicity. J. Math. Anal. Appl. 336(1), 297-315 (2007) 
3. Costakis, G., Tsirivas, N.: Doubly universal Taylor series. J. Approx. Theory 180, 21-31 (2014)

4. Müller, J., Yavrian, A.: On polynomial sequences with restricted growth near infinity. Bull. London Math. Soc. 34(2), 189-199 (2002)

5. Nestoridis, V.: Universal Taylor series. Ann. Inst. Fourier (Grenoble) 46(5), 1293-1306 (1996)

6. Nestoridis, V.: An extension of the notion of universal Taylor series. In: Papamichael, N., Ruscheweyh, S., Saff, E.B. (eds.) Computational Methods and Function Theory 1997. Series in Approximation and Decomposition, vol. 11, pp. 421-430. World Scientific, River Edge (1999)

7. Ransford, T.: Potential Theory in the Complex Plane. London Mathematical Society Student Texts, vol. 28. Cambridge University Press, Cambridge (1995) 